\title{
Spatial damping of propagating sausage waves in coronal cylinders
}

\author{
Ming-Zhe Guo ${ }^{1,2}$, Shao-Xia Chen ${ }^{1}, \mathrm{Bo} \mathrm{Li}^{1}$, Li-Dong Xia ${ }^{1}$, and Hui $\mathrm{Yu}^{1}$ \\ ${ }^{1}$ Shandong Provincial Key Laboratory of Optical Astronomy and Solar-Terrestrial Environment, and Institute of Space Sciences,
Shandong University Weihai, Weihai 264209, PR China
e-mail: bbl@sdu.edu.cn
2 CAS Key Laboratory of Geospace Environment, University of Science \& Technology of China, Chinese Academy of Sciences,
Hefei 230026, PR China
}

Received 30 May 2015 / Accepted 28 July 2015

\section{ABSTRACT}

\begin{abstract}
Context. Sausage modes are important in coronal seismology. Spatially damped propagating sausage waves were recently observed in the solar atmosphere.

Aims. We examine how wave leakage influences the spatial damping of sausage waves propagating along coronal structures modeled by a cylindrical density enhancement embedded in a uniform magnetic field.

Methods. Working in the framework of cold magnetohydrodynamics, we solve the dispersion relation (DR) governing sausage waves for complex-valued, longitudinal wavenumber $k$ at given real angular frequencies $\omega$. For validation purposes, we also provide analytical approximations to the DR in the low-frequency limit and in the vicinity of $\omega_{\mathrm{c}}$, the critical angular frequency separating trapped from leaky waves.

Results. In contrast to the standing case, propagating sausage waves are allowed for $\omega$ much lower than $\omega_{\mathrm{c}}$. However, while able to direct their energy upward, these low-frequency waves are subject to substantial spatial attenuation. The spatial damping length shows little dependence on the density contrast between the cylinder and its surroundings, and depends only weakly on frequency. This spatial damping length is of the order of the cylinder radius for $\omega \lesssim 1.5 v_{\mathrm{Ai}} / a$, where $a$ and $v_{\mathrm{Ai}}$ are the cylinder radius and the Alfvén speed in the cylinder, respectively.

Conclusions. If a coronal cylinder is perturbed by symmetric boundary drivers (e.g., granular motions) with a broadband spectrum, wave leakage efficiently filters out the low-frequency components.
\end{abstract}

Key words. magnetohydrodynamics (MHD) - Sun: corona - Sun: magnetic fields - waves

\section{Introduction}

Considerable progress has been made in coronal seismology thanks to the abundantly identified waves and oscillations in the structured solar atmosphere (for a recent review, see De Moortel \& Nakariakov 2012; and also Ballester et al. 2007; Nakariakov \& Erdélyi 2009; Erdélyi \& Goossens 2011, for three recent topical issues). Equally important is a detailed theoretical understanding of the collective wave modes supported by magnetized cylinders (e.g., Roberts 2000). While kink waves (with azimuthal wavenumber $m=1$ ) have attracted much attention since their measurements with the Transition Region And Coronal Explorer (TRACE; Nakariakov et al. 1999; Aschwanden et al. 1999), sausage waves prove important in interpreting secondscale, quasi-periodic pulsations (QPPs) in the light curves of solar flares (see Nakariakov \& Melnikov 2009, for a recent review). Their importance is strengthened given their recent detection in both the chromosphere (Morton et al. 2012) and photosphere (Dorotovič et al. 2014; Grant et al. 2015).

Standing sausage modes are well understood. For instance, two distinct regimes are known to exist, depending on the longitudinal wavenumber $k$ (Nakariakov \& Verwichte 2005). The trapped regime results when $k$ exceeds some critical value $k_{\mathrm{c}}$, whereas the leaky regime arises when the opposite is true. Both eigenmode analyses (Kopylova et al. 2007; Vasheghani Farahani et al. 2014) and numerical simulations from an initial-value-problem perspective (e.g., Nakariakov et al. 2012) indicate that the period $P$ of sausage modes increases smoothly with decreasing $k$ until reaching some $P_{0}$ in the thin-cylinder limit ( $k a \rightarrow 0$ with $a$ being the cylinder radius). Likewise, since it is identically infinite in the trapped regime, the attenuation time $\tau$ decreases with decreasing $k$ before saturating at $\tau_{0}$ when $k a=0$. Furthermore, $P_{0}$ is determined primarily by $a / v_{\mathrm{Ai}}$, where $v_{\mathrm{Ai}}$ is the Alfvén speed in the cylinder (Roberts et al. 1984), with the detailed transverse density distribution playing an important role (Nakariakov et al. 2012; Chen et al. 2015). This is why second-scale QPPs are attributed to standing sausage modes, since $a / v_{\mathrm{Ai}}$ is of the order of seconds for typical coronal structures. On the other hand, the ratio $\tau_{0} / P_{0}$ is basically proportional to the density contrast (e.g., Kopylova et al. 2007), meaning that high-quality sausage modes are associated with coronal structures with densities considerably exceeding their surroundings.

Interestingly, the dispersive behavior of trapped modes is also important in understanding impulsively generated sausage waves (Roberts et al. 1984). When measured at a distance from the source, the signals from these propagating waves possess three phases: periodic, quasi-periodic, and decay. The frequency dependence of the longitudinal group speed $v_{\text {gr }}$ is crucial in this context. In particular, whether the quasi-periodic phase exists depends on the existence of a local minimum in $v_{\mathrm{gr}}$, which in turn depends on the density profile transverse to the structure (Edwin \& Roberts 1988; Nakariakov \& Roberts 1995). This analytical expectation, extensively reproduced in numerical 
simulations (e.g., Murawski \& Roberts 1993; Selwa et al. 2004; Nakariakov et al. 2004), well explains the time signatures of the wave trains discovered with the Solar Eclipse Coronal Imaging System (Williams et al. 2001, 2002; Katsiyannis et al. 2003) as well as those measured with the Atmospheric Imaging Assembly (AIA) on board the Solar Dynamics Observatory (SDO; Yuan et al. 2013).

We intend to examine the spatial damping of leaky sausage waves propagating along coronal cylinders in response to photospheric motions due to, for example, granular convection (Berghmans et al. 1996). One reason for conducting this study is that besides the observations showing that propagating sausage waves abound in the chromosphere (Morton et al. 2012), a recent study clearly demonstrates the spatial damping of sausage waves propagating from the photosphere to the transition region in a pore (Grant et al. 2015). Another motivation is connected to the intensive interest (Terradas et al. 2010; Hood et al. 2013; Pascoe et al. 2013) in employing resonant absorption to understand the spatial damping of propagating kink waves measured with the Coronal Multi-Channel Polarimeter (CoMP) instrument (Tomczyk et al. 2007; Tomczyk \& McIntosh 2009). A leading mechanism for interpreting the temporal damping of standing kink modes (Ruderman \& Roberts 2002; Aschwanden et al. 2003, and references therein), resonant absorption is found to attenuate propagating kink waves with a spatial length inversely proportional to wave frequency (Terradas et al. 2010). If attributing the generation of these kink waves to broadband photospheric perturbations, one expects that resonant absorption essentially filters out the high-frequency components. One then naturally asks: what role does wave leakage play in attenuating propagating sausage waves? And, what is the frequency dependence of the associated damping length?

This manuscript is structured as follows. We present the necessary equations, the dispersion relation (DR) in particular, in Sect. 2, and then present our numerical solutions to the DR in Sect. 3 where we also derive two analytical approximations to the DR for validation purposes. Finally, a summary is given in Sect. 4.

\section{Problem formulation}

We consider sausage waves propagating in a structured corona modeled by a plasma cylinder with radius $a$ embedded in a uniform magnetic field $\boldsymbol{B}=B \hat{z}$, where a cylindrical coordinate system $(r, \theta, z)$ is adopted. The cylinder is directed along the $z$-direction. A piecewise constant (top hat) density profile is adopted, with the densities inside and external to the cylinder being $\rho_{\mathrm{i}}$ and $\rho_{\mathrm{e}}$, respectively $\left(\rho_{\mathrm{e}}<\rho_{\mathrm{i}}\right)$. The Alfvén speeds, $v_{\mathrm{Ai}}$ and $v_{\mathrm{Ae}}$, follow from the definition $v_{\mathrm{A}}=\sqrt{B^{2} / 4 \pi \rho}$. Appropriate for the solar corona, zero- $\beta$, ideal magnetohydrodynamic (MHD) equations are adopted. In this case, sausage waves do not perturb the $z$-component of the plasma velocity. Let $\delta v_{r}$ denote the radial velocity perturbation, and $\delta b_{r}, \delta b_{z}$ denote the radial and longitudinal components of the perturbed magnetic field $\delta \boldsymbol{b}$, respectively. The perturbed magnetic pressure, or equivalently total pressure in the zero- $\beta$ case, is then $\delta p_{\text {tot }}=B \delta b_{z} / 4 \pi$.

Let us Fourier decompose any perturbed value $\delta f(r, z ; t)$ as

$\delta f(r, z ; t)=\operatorname{Re}\{\tilde{f}(r) \exp [-\mathrm{i}(\omega t-k z)]\}$.

With the definition

$\mu_{\mathrm{i}}^{2}=\frac{\omega^{2}}{v_{\mathrm{Ai}}^{2}}-k^{2}, \quad \mu_{\mathrm{e}}^{2}=\frac{\omega^{2}}{v_{\mathrm{Ae}}^{2}}-k^{2} \quad\left(-\frac{\pi}{2}<\arg \mu_{\mathrm{i}}, \arg \mu_{\mathrm{e}} \leq \frac{\pi}{2}\right)$, linearizing the ideal MHD equations then leads to (e.g., Cally 1986)

$\frac{1}{r}\left(r \tilde{p}_{\text {tot }}^{\prime}\right)^{\prime}+\mu^{2} \tilde{p}_{\text {tot }}=0$,

where the prime ${ }^{\prime}=\mathrm{d} / \mathrm{d} r$, and this equation is valid both inside (denoted by the subscript i) and outside (the subscript e) the cylinder. The solutions to Eq. (3) are given by

$\tilde{p}_{\text {tot, } \mathrm{i}}=A_{\mathrm{i}} J_{0}\left(\mu_{\mathrm{i}} r\right), \tilde{p}_{\text {tot, } \mathrm{e}}=A_{\mathrm{e}} H_{0}^{(1)}\left(\mu_{\mathrm{e}} r\right)$,

where $J_{n}$ and $H_{n}^{(1)}$ are the $n$-th-oder Bessel and Hankel functions of the first kind, respectively (here $n=0$ ). For future reference, we also give the expressions for the Fourier amplitudes of some relevant perturbations,

$\tilde{b}_{z}=\frac{4 \pi \tilde{p}_{\mathrm{tot}}}{B}, \tilde{v}_{r}=\frac{-\mathrm{i} \omega}{\mu^{2}} \frac{\tilde{b}_{z}^{\prime}}{B}$, and $\tilde{b}_{r}=-\frac{k B}{\omega} \tilde{v}_{r}$.

In addition, the energy flux density carried by the sausage waves is given by

$\boldsymbol{F}=-\frac{1}{4 \pi}(\delta \boldsymbol{v} \times \boldsymbol{B}) \times \delta \boldsymbol{b}=\delta p_{\mathrm{tot}} \delta v_{r} \hat{r}-\frac{\delta v_{r} \delta b_{r}}{4 \pi} B \hat{z}$.

The dispersion relation (DR) for sausage waves follows from the requirements that the Fourier amplitudes of both the total pressure perturbation $\tilde{p}_{\text {tot }}$ and the radial velocity perturbation $\tilde{v}_{r}$ be continuous at the cylinder boundary $r=a$. More properly, the continuity of the Fourier amplitude of the radial Lagrangian displacement $\tilde{\xi}_{r}$ should be used in place of that of $\tilde{v}_{r}$. Even though in the static case the two requirements are equivalent given that $\tilde{v}_{r}=-\mathrm{i} \omega \tilde{\xi}_{r}$, the equivalence is not present when axial flows exist (e.g., Goossens et al. 1992; Li et al. 2014). The DR reads (e.g., Cally 1986)

$\frac{\mu_{\mathrm{i}}}{\mu_{\mathrm{e}}}=\frac{J_{1}\left(\mu_{\mathrm{i}} a\right)}{J_{0}\left(\mu_{\mathrm{i}} a\right)} \frac{H_{0}^{(1)}\left(\mu_{\mathrm{e}} a\right)}{H_{1}^{(1)}\left(\mu_{\mathrm{e}} a\right)}$.

Throughout this study, we only examine the solutions that correspond to the lowest critical longitudinal wavenumber in the trapped regime. Unless otherwise specified, we solve Eq. (7) by assuming that the angular frequency $\omega$ is real, and the longitudinal wavenumber $k$ is complex $\left(k=k_{\mathrm{R}}+\mathrm{i} k_{\mathrm{I}}\right)$. For these propagating waves, the energy flux density averaged over a wave period $2 \pi / \omega$ is

$\left\langle F_{r}\right\rangle=\frac{1}{2} \operatorname{Re}\left(\tilde{p}_{\mathrm{tot}} \tilde{U}_{r}^{*}\right) \exp \left(-2 k_{\mathrm{I}} z\right)$

$\left\langle F_{z}\right\rangle=\frac{1}{2} \operatorname{Re}\left(-\frac{B}{4 \pi} \tilde{b}_{r} \tilde{v}_{r}^{*}\right) \exp \left(-2 k_{\mathrm{I}} z\right)$

where the subscripts $r$ and $z$ denote the radial and longitudinal components, respectively. Furthermore, $\tilde{f}^{*}$ means taking the complex conjugate of some complex-valued $\tilde{f}$. (See Appendix A for a derivation of Eqs. (8) and (9).)

A comprehensive study was conducted recently by Moreels et al. (2015, hereafter M15) to work out the specific expressions for the energy and energy flux densities for both fast and slow sausage waves. That study differs from ours primarily in the objectives. To enable a calculation of the energy content of sausage waves in a variety of solar structures, M15 employed the specific expressions for the eigenfunctions and focused on trapped waves. However, the aim of the present study is to examine the spatial damping of propagating leaky waves. For this purpose, 
M.-Z. Guo et al.: Propagating sausage waves in coronal cylinders
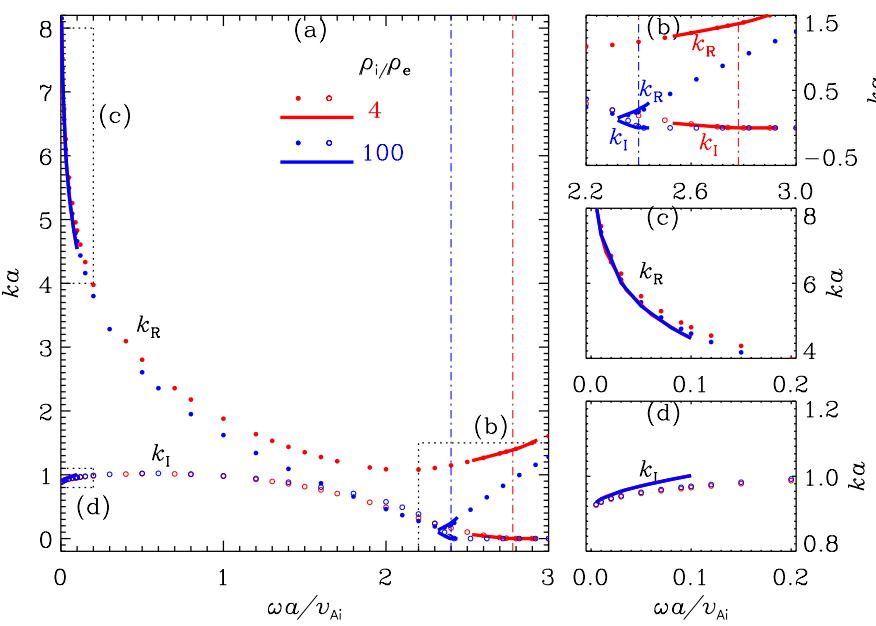

Fig. 1. Dependence on angular frequency $\omega$ of the real $\left(k_{\mathrm{R}}\right.$, the solid dots) and imaginary ( $k_{\mathrm{I}}$, open dots) parts of the longitudinal wavenumber. Two density ratios $\rho_{\mathrm{i}} / \rho_{\mathrm{e}}=4$ and 100 are examined, and are given by the symbols and curves in red and blue, respectively. The vertical dash-dotted lines correspond to the critical angular frequency $\omega_{\mathrm{c}}$, which separates the leaky (to the left of $\omega_{\mathrm{c}}$ ) from trapped (right) regimes. Figure 1a presents an overview of the dispersion curves, while Fig. 1b (Figs. 1c and d) examines the portion where $\omega$ is close to $\omega_{\mathrm{c}}(\omega$ approaches zero). The dots are found by numerically solving the full dispersion relation (DR, Eq. (7)) by assuming a real $\omega$ but a complexvalued $k=k_{\mathrm{R}}+\mathrm{i} k_{\mathrm{I}}$, while the curves represent the solutions to the approximate DR (see text for details).

the specific expressions for the energy flux densities are not necessary. Rather, we only need to evaluate the sign of $\left\langle F_{r}\right\rangle$ at large distances from the cylinder to offer a physical explanation for the spatial damping. Likewise, the sign of $\left\langle F_{z}\right\rangle$ is necessary to address whether the spatially damped waves can impart their energy upward. We would like to stress that while the energetics of leaky waves cannot be addressed with an eigenvalue problem approach (see our discussion toward the end of Sect. 3), evaluating the signs of $\left\langle F_{r}\right\rangle$ and $\left\langle F_{z}\right\rangle$ makes physical sense. A similar conclusion was reached in M15 for the trapped waves at the critical axial wavenumber, below which trapped waves are no longer allowed. Regarding the technical details, the energy flux density, Eq. (6), is equivalent to the Poynting flux given the cold MHD approximation. In other words, the contribution due to thermal pressure, given by $\langle\boldsymbol{T}\rangle$ in Eq. (4) of M15, vanishes here.

\section{Results}

Figure 1 presents the solutions to the DR for two different density ratios, one small $\left(\rho_{\mathrm{i}} / \rho_{\mathrm{e}}=4\right.$, the red curves and symbols) and the other rather large $\left(\rho_{\mathrm{i}} / \rho_{\mathrm{e}}=100\right.$, blue $)$. The real (the filled dots) and imaginary (open) parts of the longitudinal wavenumber $k$ are presented as functions of the angular frequency $\omega$. The vertical dash-dotted lines separate the trapped regime (where $k_{\mathrm{I}} \equiv 0$ ) from the leaky regime $\left(k_{\mathrm{I}} \neq 0\right.$ ), and correspond to $\omega_{\mathrm{c}}=k_{\mathrm{c}} v_{\mathrm{Ae}}$ with $k_{\mathrm{c}}=2.4048 /\left(a \sqrt{\rho_{\mathrm{i}} / \rho_{\mathrm{e}}-1}\right)$ (Nakariakov \& Verwichte 2005). Let us first examine the validity of the numerical solutions. To this end, the dispersion curves in the neighborhood of $\omega_{\mathrm{c}}$ are amplified in Fig. 1b. Assuming that $|\Delta \omega|=\left|\omega-\omega_{\mathrm{c}}\right| \ll \omega_{\mathrm{c}}$, one can derive the analytical expression,

$$
\Delta k=\frac{1+\frac{i}{\pi}\left\{\ln \left[\frac{k_{\mathrm{c}}^{2} a^{2}}{2}\left(\frac{\Delta \omega}{\omega_{\mathrm{c}}}-\frac{\Delta k}{k_{\mathrm{c}}}\right)\right]+2 \gamma-\frac{v_{\mathrm{Ae}}^{2}}{v_{\mathrm{Ai}}^{2}}\right\}}{1+\frac{i}{\pi}\left\{\ln \left[\frac{k_{\mathrm{c}}^{2} a^{2}}{2}\left(\frac{\Delta \omega}{\omega_{\mathrm{c}}}-\frac{\Delta k}{k_{\mathrm{c}}}\right)\right]+2 \gamma-1\right\}} \frac{k_{\mathrm{c}}}{\omega_{\mathrm{c}}} \Delta \omega,
$$
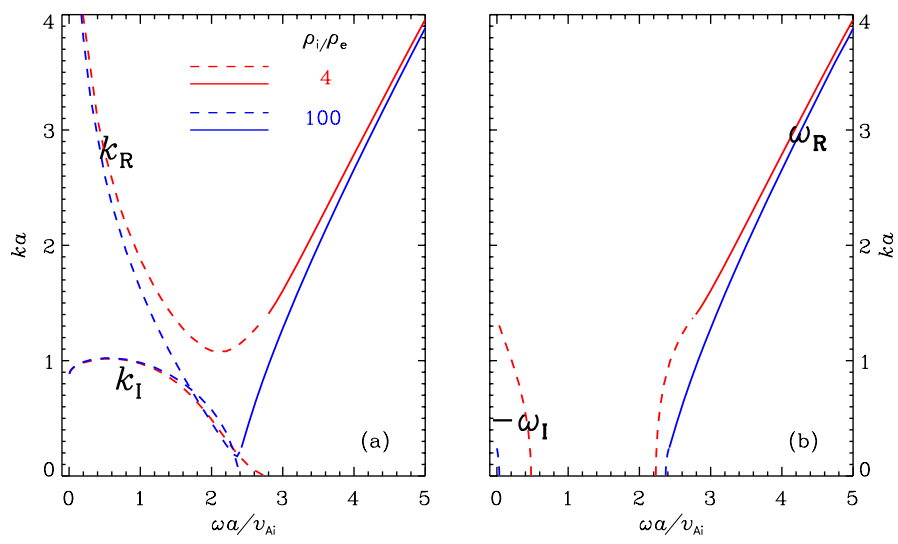

Fig. 2. Dispersion curves of a) propagating and b) standing sausage waves. In a) the real $\left(k_{\mathrm{R}}\right)$ and imaginary $\left(k_{\mathrm{I}}\right)$ parts of the longitudinal wavenumber are given as functions of the real angular frequency $\omega$. In b) the real $\left(\omega_{\mathrm{R}}\right)$ and imaginary $\left(\omega_{\mathrm{I}}\right)$ parts of $\omega$ are shown as functions of the real wavenumber $k$, which is given by the vertical axis though. In both panels, the solid (dashed) curves represent the trapped (leaky) regime. Two density ratios $\left(\rho_{\mathrm{i}} / \rho_{\mathrm{e}}=4\right.$ and 100) are examined as represented by the red and blue curves, respectively.

where $\Delta k=k-k_{\mathrm{c}}$. Interestingly, Eq. (10) agrees with Eq. (16) in Vasheghani Farahani et al. (2014) even though standing modes with real $k$ and complex-valued $\omega$ are examined there. The primary difference between the two is the appearance of the Euler constant $\gamma=0.577$, which we found is necessary to retain when the logarithmic term does not substantially exceed unity. In Fig. 1b, the solid curves represent the solutions to Eq. (10). These well approximate the solutions to the full DR given by the dots in both trapped and leaky regimes.

Another portion of the dispersion curves, which is analytically tractable, is when $\omega \rightarrow 0$. In this case, the DR can be approximated by

$\mu_{\mathrm{i}} a=\arctan \left(\frac{\mu_{\mathrm{e}}-\mathrm{i} \mu_{\mathrm{i}}}{\mu_{\mathrm{e}}+\mathrm{i} \mu_{\mathrm{i}}}\right)$.

To arrive at Eq. (11), we have assumed that $\left|\mu_{\mathrm{i}} a\right|,\left|\mu_{\mathrm{e}} a\right| \gg 1$, which can be justified a posteri. The real (imaginary) part of the solution to Eq. (11) is presented by the curves in Fig. 1c (Fig. 1d), and is found to agree well with the solutions to the full DR, represented by the dots. It is also clear that in the lowfrequency portion the solutions to the DR for the two density ratios are close to each other, despite that the ratios differ substantially. This is understandable from Eqs. (11) and (2), because $\mu_{\mathrm{i}}^{2}$ and $\mu_{\mathrm{e}}^{2}$ both approach $-k^{2}$ when $\omega$ approaches zero, therefore they have little dependence on the Alfvén speeds or densities.

Figure $2 \mathrm{a}$ presents once again the dispersion curves for propagating sausage waves, where the real and imaginary parts of $k$ are given as functions of $\omega$. For comparison, Fig. 2b presents the dispersion curves for standing waves, obtained by solving Eq. (7) for complex-valued $\omega\left(\omega=\omega_{\mathrm{R}}+i \omega_{\mathrm{I}}\right)$ at given real values of $k$. Note that here $k$ is given by the vertical axis, and $-\omega_{\text {I }}$ is plotted instead of $\omega_{\text {I }}$ because $\omega_{\text {I }} \leq 0$. In both cases, we examine two density ratios with $\rho_{\mathrm{i}} / \rho_{\mathrm{e}}$ being 4 (the red curves) and 100 (blue), and present the trapped (leaky) regime with the solid (dashed) curves. One can see that in the trapped regime, Figs. $2 \mathrm{a}$ and $\mathrm{b}$ agree exactly with one another, as expected for the situation where both $\omega$ and $k$ are real. In the trapped regime, however, some distinct differences appear. For standing waves, with decreasing $k$, the real (imaginary) part of the angular frequency $\omega_{\mathrm{R}}\left(\omega_{\mathrm{I}}\right)$ decreases (increases in magnitude) and saturates 
when $k$ approaches zero. This $k$-dependence of both $\omega_{\mathrm{R}}$ and $\omega_{\text {I }}$ is well understood (e.g., Kopylova et al. 2007; Nakariakov et al. 2012; Vasheghani Farahani et al. 2014). It suffices to note that the temporal damping clearly depends on $\rho_{\mathrm{i}} / \rho_{\mathrm{e}}$ : the higher the density ratio, the slower the temporal damping. In fact, for large $\rho_{\mathrm{i}} / \rho_{\mathrm{e}}$, a simple but reasonably accurate expression exists, namely, $\left|\omega_{\mathrm{I}}\right| / \omega_{\mathrm{R}} \approx(\pi / 2)\left(\rho_{\mathrm{e}} / \rho_{\mathrm{i}}\right)$ (Kopylova et al. 2007). When examining propagating waves, Fig. 2a shows that the DR permits waves with $\omega$ much lower than $\omega_{\mathrm{c}}$, in contrast to the standing case. In addition, for $\omega \lesssim v_{\mathrm{Ai}} / a$, neither $k_{\mathrm{R}}$ nor $k_{\mathrm{I}}$ shows a significant dependence on the density ratio. This is particularly true for $k_{\mathrm{I}}$, and has been explained in view of Eq. (11).

Why should the results be different if one simply changes from one perspective, where a DR is solved for complex $\omega$ as a function of real $k$, to another standpoint where the same DR is solved for complex $k$ as a function of real $\omega$ ? As has been discussed in detail by Tagger et al. (1995, hereafter TFS95), indeed the two perspectives have a close relationship when wave attenuation is weak or absent. However, some considerable difference between the two may result when strong attenuation takes place. An example of this can be found in TFS95 where the authors examined linear Alfvén waves in a partially ionized gas where ions and neutrals are imperfectly coupled. Solving the relevant DR for complex $\omega$ at real $k$ yields that $\omega$ may be purely imaginary in certain ranges of $k$. In other words, the waves may be overdamped and nonpropagating. In contrast, solving the same DR for complex $k$ at real $\omega$ yields that the real part of $k$ is always nonzero, meaning that the waves are always propagating. This led TFS95 to conclude that how to choose a perspective depends on how the waves are excited. One chooses complex $k$ and real $\omega$ to examine the spatial variation of the waves excited at a given location with a given real frequency. On the other hand, one chooses complex $\omega$ and real $k$ to follow the temporal variation of the waves in response to perturbations initially imposed in a coherent way over many wavelengths. These discussions are also valid if one examines the differences in Figs. 2a and b. In particular, the absence of standing waves with frequencies below a certain value (see Fig. 2b) can be understood given that in view of Fig. 2a, an initial perturbation cannot stay coherent in a longitudinal spatial range spanning many wavelengths and, hence, many damping lengths. Instead, the system selects frequencies and damping rates corresponding to the spatial periods externally enforced.

Let us now focus on Fig. 2a, where one can see that for both density ratios, once in the leaky regime, $k_{\mathrm{I}}$ increases with decreasing $\omega$ and somehow levels off when $\omega \lesssim 1.5 v_{\text {Ai }} / a$. On the other hand, $k_{\mathrm{R}}$ decreases when $\omega$ decreases from $\omega_{\mathrm{c}}$ before increasing monotonically when $\omega$ further decreases. Let $\omega_{\mathrm{m}}$ denote the point where $k_{\mathrm{R}}$ reaches a local minimum. It then follows that the apparent group speed $\mathrm{d} \omega / \mathrm{d} k_{\mathrm{R}} \leq 0$ for $\omega \leq \omega_{\mathrm{m}}$. One may question whether the sausage waves in this low-frequency portion can impart energy upward. As discussed in Brillouin (1960, chapter V), when waves are heavily damped, the apparent group velocity may not represent the velocity at which energy propagates. In this case, we may directly use Eq. (9) to evaluate the $z$-component of the wave energy flux density. In view of Eq. (5), one finds that

$\left\langle F_{z}\right\rangle=\frac{B^{2}}{8 \pi}\left|\tilde{v}_{r}\right|^{2}\left(\frac{k_{\mathrm{R}}}{\omega}\right) \exp \left(-2 k_{\mathrm{I}} z\right)$,

which is positive for positive $k_{\mathrm{R}} / \omega$, meaning that the lowfrequency waves in question can still direct their energy upward. However, in this case the plasma cylinder is such an inefficient waveguide that the wave energy is attenuated over a longitudinal distance of approximately the cylinder radius.

More insights can be gained by further comparing the dispersive behavior of leaky standing and propagating sausage waves as given in Fig. 2. First, for standing waves with real $k$ and complex $\omega$ (propagating waves with real $\omega$ and complex $k$ ) the radial energy flux density in the external medium, when averaged over a longitudinal wavelength $2 \pi / k$ (a wave period $2 \pi / \omega$ ), evaluates to,

$\left\langle F_{r}\right\rangle=\frac{2 \pi}{B^{2}}\left|\tilde{p}_{\text {tot }, \mathrm{e}}\right|^{2} \operatorname{Re}\left(\frac{-\mathrm{i} \omega}{\mu_{\mathrm{e}}^{2}} \frac{\tilde{p}_{\text {tot,e }}^{\prime}}{\tilde{p}_{\text {tot, } \mathrm{e}}}\right) \begin{cases}\mathrm{e}^{2 \omega_{\mathrm{I}} t} & \text { standing } \\ \mathrm{e}^{-2 k_{\mathrm{I}} z} & \text { propagating. }\end{cases}$

We note that $\left\langle F_{r}\right\rangle$ for standing waves was originally derived in Cally (1986, Eq. (3.2)). See Appendix A for a derivation of Eq. (12). Whether the waves are standing or propagating, $\tilde{p}_{\text {tot, }} \propto H_{0}^{(1)}\left(\mu_{\mathrm{e}} r\right)$ at large distances can be approximated by

$\tilde{p}_{\text {tot }, \mathrm{e}} \propto \sqrt{\frac{2}{\pi \mu_{\mathrm{e}} r}} \exp \left[\mathrm{i}\left(\mu_{\mathrm{e}} r-\frac{\pi}{4}\right)\right]$,

resulting in $\tilde{p}_{\text {tot,e }}^{\prime} / \tilde{p}_{\text {tot,e }} \approx \mathrm{i} \mu_{\mathrm{e}}$. It then follows that

$\left\langle F_{r}\right\rangle \approx \frac{2 \pi}{B^{2}}\left|\tilde{p}_{\text {tot, }}\right|^{2} \operatorname{Re}\left(\frac{\omega}{\mu_{\mathrm{e}}}\right) \begin{cases}\mathrm{e}^{2 \omega_{\mathrm{I}} t} & \text { standing } \\ \mathrm{e}^{-2 k_{\mathrm{I}} z} & \text { propagating. }\end{cases}$

From Fig. $2 b$ one can find that $\operatorname{Im}\left(\mu_{\mathrm{e}}\right)<0$ for leaky standing modes, meaning that $\left|\tilde{p}_{\text {tot,e }}\right|$ tends to grow exponentially (Eq. (13), barring the $r^{-1 / 2}$-dependence) and an eigenmode analysis does not allow us to examine the energetics of the system. However, this does not mean that this analysis is physically irrelevant because what matters is that the apparent temporal damping can be accounted for by the outwardly going energy flux density $\left(\left\langle F_{r}\right\rangle>0\right.$ because $\operatorname{Re}\left(\omega / \mu_{\mathrm{e}}\right)>0$, see Eq. (14)). Indeed, numerical studies starting with initial standing waves suggest that the temporal damping after a transient stage exactly matches the attenuation rate given by the eigenmode analysis (Terradas et al. 2007). For propagating waves, one finds from Fig. 2a that once again $\operatorname{Im}\left(\mu_{\mathrm{e}}\right)<0$ and $\operatorname{Re}\left(\omega / \mu_{\mathrm{e}}\right)>0$. Hence, similar to the standing case, an eigenmode analysis does not permit an investigation into the energetics of propagating waves. However, if a coronal structure is perturbed with a harmonic boundary driver, one expects to see that the apparent spatial damping after some transient phase is given by the attenuation length $1 / k_{\mathrm{I}}$ obtained from this eigenmode analysis. And this attenuation is once again associated with the outwardly directed $\left\langle F_{r}\right\rangle$. This expectation can be readily numerically tested, in much the same way that the expected spatial damping of kink waves due to resonant absorption was tested (Pascoe et al. 2013).

\section{Summary}

This study is motivated by the apparent lack of a dedicated study on the role that wave leakage plays in spatially attenuating propagating sausage waves supported by density-enhanced cylinders in the corona. To this end, we worked in the framework of cold MHD, and numerically solved the dispersion relation (DR, Eq. (7)) for complex-valued longitudinal wavenumbers $k=k_{\mathrm{R}}+\mathrm{i} k_{\mathrm{I}}$ at given real angular frequencies $\omega$. To validate our numerical results, we also provided the analytical approximations to the full DR in the low-frequency limit $\omega \rightarrow 0$, and in the neighborhood of $\omega_{c}$, the critical angular frequency separating trapped from leaky waves. Our solutions indicate that 
while sausage waves can propagate for $\omega<\omega_{\mathrm{c}}$ and can direct their energy upward, they suffer substantial spatial attenuation. The attenuation length $\left(1 / k_{\mathrm{I}}\right)$ is approximately cylinder radius $a$ and shows little dependence on frequency or the density contrast between a coronal structure and its surroundings for $\omega \lesssim 1.5 v_{\mathrm{Ai}} / a$, where $v_{\mathrm{Ai}}$ is the Alfvén speed in the cylinder. This means that when a coronal cylinder is subject to boundary perturbations with a broadband spectrum (e.g., granular motions), wave leakage removes the low-frequency components rather efficiently. A comparison with the solutions to the DR for standing waves (real $k$, complex $\omega$ ) indicates that a close relationship between propagating and standing waves exists only when the waves are trapped or weakly damped. In addition, while an eigenmode analysis does not allow an investigation into the energetics of propagating waves, the attenuation length is expected to play an essential role in numerical simulations where coronal structures are perturbed by harmonic boundary drivers.

Acknowledgements. This research is supported by the 973 program 2012CB825601, National Natural Science Foundation of China (41174154, 41274176, 41274178, and 41474149), the Provincial Natural Science Foundation of Shandong via Grant JQ201212, and also by a special fund of Key Laboratory of Chinese Academy of Sciences.

\section{Appendix A: A derivation of the averaged energy flux densities}

This section offers a derivation of the averaged energy flux densities given in Eqs. (8), (9), and (12), following a procedure similar to that adopted by $\mathrm{Li} \& \mathrm{Li}(2007$, p. 1225). Let us first consider standing waves for which the axial wavenumber $k$ is real, but the angular frequency $\omega$ is allowed to be complex-valued $\left(\omega=\omega_{\mathrm{R}}+\mathrm{i} \omega_{\mathrm{I}}\right)$. Evaluating a perturbation $\delta f(r, z ; t)$ with Eq. (1) at given values of $[r, t]$ yields

$\delta f(r, z ; t)=\operatorname{Re}\{\breve{f} \exp (\mathrm{i} k z)\}$,

where

$\breve{f}=\tilde{f}(r) \exp (-\mathrm{i} \omega t)=\tilde{f}(r) \exp \left(-\mathrm{i} \omega_{\mathrm{R}} t\right) \mathrm{e}^{\omega_{\mathrm{I}} t}$.

With another perturbation $\delta g(r, z ; t)$ in the same form, one finds that the product $\delta f \delta g$ averaged over a wavelength $\lambda=2 \pi / k$ is

$\langle\delta f \delta g\rangle(r, t) \equiv \frac{1}{\lambda} \int_{0}^{\lambda} \delta f(r, z ; t) \delta g(r, z ; t) \mathrm{d} z$

$=\frac{1}{\lambda} \int_{0}^{\lambda} \operatorname{Re}\{\breve{f} \exp (\mathrm{i} k z)\} \operatorname{Re}\{\breve{g} \exp (\mathrm{i} k z)\} \mathrm{d} z$

$=\frac{1}{\lambda} \int_{0}^{\lambda}\left[\breve{f}_{\mathrm{R}} \cos (k z)-\breve{f}_{\mathrm{I}} \sin (k z)\right]\left[\breve{g}_{\mathrm{R}} \cos (k z)-\breve{g}_{\mathrm{I}} \sin (k z)\right] \mathrm{d} z$

$=\frac{1}{\lambda} \int_{0}^{\lambda}\left[\breve{f}_{\mathrm{R}} \breve{g}_{\mathrm{R}} \cos ^{2}(k z)+\breve{f}_{\mathrm{I}} \breve{g}_{\mathrm{I}} \sin ^{2}(k z)\right.$

$$
\left.-\left(\breve{f}_{\mathrm{R}} \breve{g}_{\mathrm{I}}+\breve{f}_{\mathrm{I}} \breve{g}_{\mathrm{R}}\right) \cos (k z) \sin (k z)\right] \mathrm{d} z
$$

$=\frac{1}{2}\left(\breve{f}_{\mathrm{R}} \breve{g}_{\mathrm{R}}+\breve{f}_{\mathrm{I}} \breve{g}_{\mathrm{I}}\right)$

$=\frac{1}{2} \operatorname{Re}\left(\breve{f}^{*} \breve{g}^{*}\right)=\frac{1}{2} \operatorname{Re}\left(\breve{f}^{*} \breve{g}\right)$,

where we have used the shorthand notations $\breve{f}_{\mathrm{R}}=\operatorname{Re} \breve{f}$ and $\breve{f}_{\mathrm{I}}=\operatorname{Im} \breve{f}$. By noting that $\breve{f}$ is expressible in terms of $\tilde{f}$ through Eq. (A2), one finds that

$\langle\delta f \delta g\rangle(r, t)=\frac{1}{2} \operatorname{Re}\left[\tilde{f}(r) \tilde{g}^{*}(r)\right] \mathrm{e}^{2 \omega_{\mathrm{I}} t}=\frac{1}{2} \operatorname{Re}\left[\tilde{f}^{*}(r) \tilde{g}(r)\right] \mathrm{e}^{2 \omega_{\mathrm{I}} t}$.
Now the energy flux density averaged over a wavelength can be evaluated with its definition, Eq. (6), the result yields

$$
\begin{aligned}
& \left\langle F_{r}\right\rangle=\frac{1}{2} \operatorname{Re}\left(\tilde{p}_{\text {tot }} \tilde{v}_{r}^{*}\right) \mathrm{e}^{2 \omega_{\mathrm{I}} t}=\frac{1}{2} \operatorname{Re}\left(\tilde{p}_{\text {tot }}^{*} \tilde{v}_{r}\right) \mathrm{e}^{2 \omega_{\mathrm{I}} t}, \\
& \left\langle F_{z}\right\rangle=\frac{1}{2} \operatorname{Re}\left(-\frac{B}{4 \pi} \tilde{b}_{r} \tilde{v}_{r}^{*}\right) \mathrm{e}^{2 \omega_{\mathrm{I}} t} .
\end{aligned}
$$

Furthermore, by noting that Eq. (5) allows $\tilde{v}_{r}$ to be expressed by

$\tilde{v}_{r}=-\mathrm{i} \frac{\omega}{\mu^{2}} \frac{4 \pi \tilde{p}_{\text {tot }}^{\prime}}{B^{2}}$

one finds that

$$
\begin{aligned}
\left\langle F_{r}\right\rangle & =\frac{2 \pi}{B^{2}} \operatorname{Re}\left[\tilde{p}_{\text {tot }}^{*}\left(-\mathrm{i} \frac{\omega}{\mu^{2}} \tilde{p}_{\text {tot }}^{\prime}\right)\right] \mathrm{e}^{2 \omega_{1} t}, \\
& =\frac{2 \pi}{B^{2}} \operatorname{Re}\left[\tilde{p}_{\text {tot }}^{*} \tilde{p}_{\text {tot }}\left(-\mathrm{i} \frac{\omega}{\mu^{2}} \frac{\tilde{p}_{\text {tot }}^{\prime}}{\tilde{p}_{\text {tot }}}\right)\right] \mathrm{e}^{2 \omega_{1} t}, \\
& =\frac{2 \pi\left|\tilde{p}_{\text {tot }}\right|^{2}}{B^{2}} \operatorname{Re}\left(-\mathrm{i} \frac{\omega}{\mu^{2}} \frac{\tilde{p}_{\text {tot }}^{\prime}}{\tilde{p}_{\text {tot }}}\right) \mathrm{e}^{2 \omega_{\mathrm{I}} t}
\end{aligned}
$$

This expression is valid both in and outside the cylinder. When applied to the external medium, it results in the first expression in Eq. (12).

Now consider propagating waves for which $\omega$ is real, whereas $k$ is allowed to be complex-valued $\left(k=k_{\mathrm{R}}+\mathrm{i} k_{\mathrm{I}}\right)$. In this case evaluating a perturbation $\delta f(r, z ; t)$ with Eq. (1) at given values of $[r, z]$ yields that

$\delta f(r, z ; t)=\operatorname{Re}\{\breve{f} \exp (-\mathrm{i} \omega t)\}$,

where

$\breve{f}=\tilde{f}(r) \exp (\mathrm{i} k z)=\tilde{f}(r) \exp \left(\mathrm{i} k_{\mathrm{R}} z\right) \mathrm{e}^{-k_{\mathrm{I}} z}$.

To evaluate the product $\delta f \delta g$ averaged over a wave period $T=$ $2 \pi / \omega$, one can follow the same procedure as in Eq. (A3) by replacing $k z$ with $-\omega t$, the result yields

$\langle\delta f \delta g\rangle(r, z) \equiv \frac{1}{T} \int_{0}^{T} \delta f(r, z ; t) \delta g(r, z ; t) \mathrm{d} t$

$=\frac{1}{2} \operatorname{Re}\left(\breve{f} \breve{g}^{*}\right)=\frac{1}{2} \operatorname{Re}\left(\breve{f}^{*} \breve{g}\right)$.

With the aid of Eq. (A10), which relates $\breve{f}$ to $\tilde{f}$, one finds that

$$
\begin{aligned}
\langle\delta f \delta g\rangle(r, z) & =\frac{1}{2} \operatorname{Re}\left[\tilde{f}(r) \tilde{g}^{*}(r)\right] \mathrm{e}^{-2 k_{1} z} \\
& =\frac{1}{2} \operatorname{Re}\left[\tilde{f}^{*}(r) \tilde{g}(r)\right] \mathrm{e}^{-2 k_{1} z} .
\end{aligned}
$$

The energy flux density averaged over a period follows from the definition, Eq. (6), and the results are given by Eqs. (8) and (9). The second expression in Eq. (12), appropriate for propagating waves, can be derived in view of Eq. (A7).

\section{References}

Aschwanden, M. J., Fletcher, L., Schrijver, C. J., \& Alexander, D. 1999, ApJ, 520,880

Aschwanden, M. J., Nightingale, R. W., Andries, J., Goossens, M., \& Van Doorsselaere, T. 2003, ApJ, 598, 1375

Ballester, J. L., Erdélyi, R., Hood, A. W., Leibacher, J. W., \& Nakariakov, V. M. 2007, Sol. Phys., 246, 1 
Berghmans, D., de Bruyne, P., \& Goossens, M. 1996, ApJ, 472, 398 Brillouin, L. 1960, in Wave Propagation and Group Velocity (New York: Academic Press)

Cally, P. S. 1986, Sol. Phys., 103, 277

Chen, S.-X., Li, B., Xia, L.-D., \& Yu, H. 2015, Sol. Phys., DOI: $10.1007 / \mathrm{s} 11207-015-0751-7$

De Moortel, I., \& Nakariakov, V. M. 2012, Roy. Soc. Lond. Philos. Trans. Ser. A, 370,3193

Dorotovič, I., Erdélyi, R., Freij, N., Karlovský, V., \& Márquez, I. 2014, A\&A, 563, A12

Edwin, P. M., \& Roberts, B. 1988, A\&A, 192, 343

Erdélyi, R., \& Goossens, M. 2011, Space Sci. Rev., 158, 167

Goossens, M., Hollweg, J. V., \& Sakurai, T. 1992, Sol. Phys., 138, 233

Grant, S. D. T., Jess, D. B., Moreels, M. G., et al. 2015, ApJ, 806, 132

Hood, A. W., Ruderman, M., Pascoe, D. J., et al. 2013, A\&A, 551, A39

Katsiyannis, A. C., Williams, D. R., McAteer, R. T. J., et al. 2003, A\&A, 406, 709

Kopylova, Y. G., Melnikov, A. V., Stepanov, A. V., Tsap, Y. T., \& Goldvarg, T. B. 2007, Astron. Lett., 33, 706

Li, B., \& Li, X. 2007, ApJ, 661, 1222

Li, B., Chen, S.-X., Xia, L.-D., \& Yu, H. 2014, A\&A, 568, A31

Moreels, M. G., Van Doorsselaere, T., Grant, S. D. T., Jess, D. B., \& Goossens, M. 2015, A\&A, 578, A60

Morton, R. J., Verth, G., Jess, D. B., et al. 2012, Nat. Commun., 3, 1315

Murawski, K., \& Roberts, B. 1993, Sol. Phys., 144, 101
Nakariakov, V. M., \& Erdélyi, R. 2009, Space Sci. Rev., 149, 1

Nakariakov, V. M., \& Melnikov, V. F. 2009, Space Sci. Rev., 149, 119

Nakariakov, V. M., \& Roberts, B. 1995, Sol. Phys., 159, 399

Nakariakov, V. M., \& Verwichte, E. 2005, Liv. Rev. Sol. Phys., 2, 3

Nakariakov, V. M., Ofman, L., Deluca, E. E., Roberts, B., \& Davila, J. M. 1999, Science, 285,862

Nakariakov, V. M., Arber, T. D., Ault, C. E., et al. 2004, MNRAS, 349, 705

Nakariakov, V. M., Hornsey, C., \& Melnikov, V. F. 2012, ApJ, 761, 134

Pascoe, D. J., Hood, A. W., De Moortel, I., \& Wright, A. N. 2013, A\&A, 551, A40

Roberts, B. 2000, Sol. Phys., 193, 139

Roberts, B., Edwin, P. M., \& Benz, A. O. 1984, ApJ, 279, 857

Ruderman, M. S., \& Roberts, B. 2002, ApJ, 577, 475

Selwa, M., Murawski, K., \& Kowal, G. 2004, A\&A, 422, 1067

Tagger, M., Falgarone, E., \& Shukurov, A. 1995, A\&A, 299, 940

Terradas, J., Andries, J., \& Goossens, M. 2007, Sol. Phys., 246, 231

Terradas, J., Goossens, M., \& Verth, G. 2010, A\&A, 524, A23

Tomczyk, S., \& McIntosh, S. W. 2009, ApJ, 697, 1384

Tomczyk, S., McIntosh, S. W., Keil, S. L., et al. 2007, Science, 317, 1192

Vasheghani Farahani, S., Hornsey, C., Van Doorsselaere, T., \& Goossens, M. 2014, ApJ, 781, 92

Williams, D. R., Phillips, K. J. H., Rudawy, P., et al. 2001, MNRAS, 326, 428

Williams, D. R., Mathioudakis, M., Gallagher, P. T., et al. 2002, MNRAS, 336, 747

Yuan, D., Shen, Y., Liu, Y., et al. 2013, A\&A, 554, A144 